\title{
Vehicle Carrying Safety Assessment for Reinforced Concrete Grillage Bridge in Combined Bending, Torsion and Shear
}

\author{
aYue An, ${ }^{\text {bZhi Sun }}$ \\ aDepartment of Bridge Engineering, Tongji University, Shanghai, China \\ bState Key Laboratory on Disaster Reduction in Civil Engineering, Shanghai, China
}

KEYWORD: RC grillage bridge, torsion and shear interaction, safety, equilibrium plasticity truss model

ABSTRACT: Reinforced concrete simply-supported grillage girder bridges are of multiple possible failure modes considering torsion and shear interactions. Equilibrium plasticity truss model was adopted to search for the first cross-section failure mode of the bridge structure. The component resistances were calculated according to Chinese JTG D62-2004 code, US ACI 318-11 code, and the equilibrium plastic truss model. The comparison shows that the consideration of torsion and shear will reduce the capacity of bridge components. Stirrup spacing was adjusted to control the failure mode to be bending dominated failure. Sensitivity analysis was performed and the results indicate that effective depth of girder is the most sensitive parameter to influence load carrying safety of the studied bridge considering the interaction of bending, torsion and shear.

\section{INTRODUCTION}

During the operation of reinforced concrete $(\mathrm{RC})$ grillage highway bridges, the failure of a normal or skew cross-section means that bridge cannot offer load carrying capacity as designed any longer. Structure resistance corresponding to the first-passage cross-section failure is thus an important threshold to ensure the safety of bridge structure. Although the simply-supported RC grillage bridges are dominated by their bending behavior (Zhang \& Sun 2014), the combined action of torsion and shear will induce the reduction of bending capacity or uncontrollable shear or torsion dominated failures.

In the calculation of resistance of RC component, the design codes currently in duty take the consideration that shear stresses caused by shear and torsion are coupled in some way to modify the resistance formula of shear and torsion. The coupling between bending, torsion and shear is not considered. The coupled action of bending, torsion and shear (B-T-S) are still a research topic. Elfgen (1972) made the assumption that stirrup and bending rebar yields at the same time of the crosssection limit states and derived the interaction equation for the plastic failure modes of RC component with rectangular cross-section. Ewida \& McMullen (1981) studied B-T-S interaction relationships of RC T- members with different volume of the stirrups. They observed an up-to 43\% strength increase of the member in combined loading. Hsu \& Mo (2010) summarized the interaction relationships of RC components with box section considering B-T-S interaction. They pointed out that if the compatibility condition is considered, some of the steel may not yield and the predicted capacity may not be reached.

This paper investigates the first-passage cross-section failure of a typical RC grillage highway bridge based on the equilibrium plasticity truss (EPT) model. Comparison of resistance results according to Chinese JTG D62-2004 Code, US ACI 318-11 Code and EPT model is carried out. The stirrup spacing adjustment measure to control the first cross-section failure as a bending dominated failure is discussed. At last, structural parameters sensitivity analysis is conducted to study their influence on vehicle carrying capacity of this bridge.

\section{EQUILIBRIUM PLASTICITY TRUSS MODEL}

Given an ideal rectangular section with longitudinal rebars in top and bottom corners and stirrups with spacing $s$ (Fig. 1), the yielding stress of rebars at top corners, bottom corners, and stirrups are 
denoted as $\sigma_{1 t}^{y}, \sigma_{1 b}^{y}$, and $\sigma_{w}^{y}$ respectively. Subjected to pure torsion, RC beam will crack and form pieces of compressive inclined columns. According to torsion mechanism, the torsion is mainly bear by outside concrete. The concrete beam is equivalently modeled as a tube with a single rebar in corners. The height and width of the beam is defined as $h$ and $b$. The distances between the legs of stirrup are $h^{\prime}$ and $b^{\prime}$. The stirrup spacing is $s$. The torsion acting on concrete beam is balanced by moments caused by forces on inclined concrete columns.

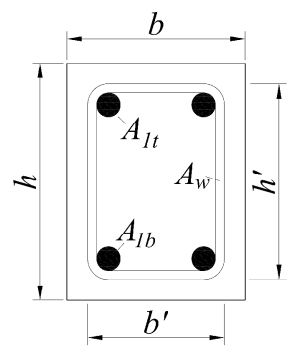

Figure 1. A typical analog truss cross-section

When it comes to B-T-S interaction, the distribution of principal tensile stress along the walls of tube is the superposition of the correspondents of pure shear and pure torsion. Analog space truss is made up of longitudinal bars, stirrups and compressive inclined concrete columns resulting from cracks. Three typical failure modes can be derived on stress resultant level based on the EPT model. They are bending dominated failure (mode $\mathrm{t}$ ), torsional dominated failure (mode $\mathrm{c}$ ) and sheartorsion dominated failure (mode $s$ ). The corresponding yield functions and resistances formula are presented below.

\section{Mode t: bending dominated failure}

Yielding equation:

$$
\frac{M}{M_{R}^{t}}+\left(\frac{T}{T_{R}^{t}}\right)^{2}+\left(\frac{V}{V_{R}^{t}}\right)^{2}=1
$$

Bending resistance:

$$
M_{R}^{t}=2 A_{1 b} \sigma_{1 b}^{y} h^{\prime}
$$

Torsional resistance:

$$
T_{R}^{t}=2 b^{\prime} h^{\prime} \frac{A_{w} \sigma_{w}^{y}}{s} \sqrt{\frac{2 A_{1 b} \sigma_{1 b}^{y}}{b^{\prime}+h^{\prime}} \cdot \frac{s}{A_{w} \sigma_{w}^{y}}}
$$

Shear resistance:

$$
V_{R}^{t}=2 h^{\prime} \frac{A_{w} \sigma_{w}^{y}}{s} \sqrt{\frac{2 A_{1 b} \sigma_{1 b}^{y}}{h^{\prime}} \cdot \frac{s}{A_{w} \sigma_{w}^{y}}}
$$

\section{Mode c: torsion dominated failure}

Yielding equation:

$$
-\frac{M}{M_{R}^{c}}+\left(\frac{T}{T_{R}^{c}}\right)^{2}+\left(\frac{V}{V_{R}^{c}}\right)^{2}=1
$$

Bending resistance:

$$
M_{R}^{c}=-2 F_{1 t}^{y} h^{\prime}=-2 A_{1 t} \sigma_{1 t}^{y} h^{\prime}
$$

Torsional resistance:

$$
T_{R}^{c}=2 b^{\prime} h^{\prime} \frac{A_{w} \sigma_{w}^{y}}{s} \sqrt{\frac{2 A_{1 t} \sigma_{1 t}^{y}}{b^{\prime}+h^{\prime}} \cdot \frac{s}{A_{w} \sigma_{w}^{y}}}
$$

Shear resistance: 


$$
V_{R}^{c}=2 h^{\prime} \frac{A_{w} \sigma_{w}^{y}}{s} \sqrt{\frac{2 A_{1 t} \sigma_{1 t}^{y}}{h^{\prime}} \cdot \frac{s}{A_{w} \sigma_{w}^{y}}}
$$

\section{Mode s: shear-torsion dominated failure}

Yielding equation:

$\left(\frac{T}{T_{R}^{s}}\right)^{2}+\left(\frac{V}{V_{R}^{s}}\right)^{2}+\frac{T V}{T_{R}^{s} V_{R}^{s}} \frac{2}{\sqrt{1+b^{\prime} / h^{\prime}}}=1$

Torsional resistance:

$$
T_{R}^{s}=2 b^{\prime} h^{\prime} \frac{A_{w} \sigma_{w}^{y}}{s} \sqrt{\frac{A_{1 t} \sigma_{1 t}^{y}+A_{1 b} \sigma_{1 b}^{y}}{b^{\prime}+h^{\prime}} \cdot \frac{s}{A_{w} \sigma_{w}^{y}}}
$$

Shear resistance:

$$
V_{R}^{s}=2 h^{\prime} \cdot \frac{A_{w} \sigma_{w}^{y}}{s} \sqrt{\frac{A_{1 t} \sigma_{1 t}^{y}+A_{1 b} \sigma_{1 b}^{y}}{h^{\prime}} \cdot \frac{s}{A_{w} \sigma_{w}^{y}}}
$$

\section{BRIDGE AND LOAD MODEL}

The bridge considered in this paper is a simply supported RC grillage bridge, composed of 5 girders and 3 diaphragms as shown in Figure 2. The designed bridge deck width is $7 \mathrm{~m}$ and sidewalks are set up with the width of $0.75 \mathrm{~m}$. The designed vehicle load is highway Class-II vehicle load. The deck cushion is C25 concrete with an average thickness of $9 \mathrm{~cm}$, over which is $2 \mathrm{~cm}$ asphalt concrete. The girder is fabricated using concrete C30 and rebar HRB335. HPB235 is used for stirrup with a type of close hoop. The thickness of the protective layer for girder concrete is $3 \mathrm{~cm}$. The stirrup spacing is $100 \mathrm{~mm}$ near the bearings and $200 \mathrm{~mm}$ for other girder segments. The effective width for diaphragm is $183 \mathrm{~cm}$ as shown in Figure 3, which has a uniform stirrup spacing $150 \mathrm{~mm}$. The resistance provided by the web horizontal rebar is not accounted as a conservation of safety (Xu et al. 2014).

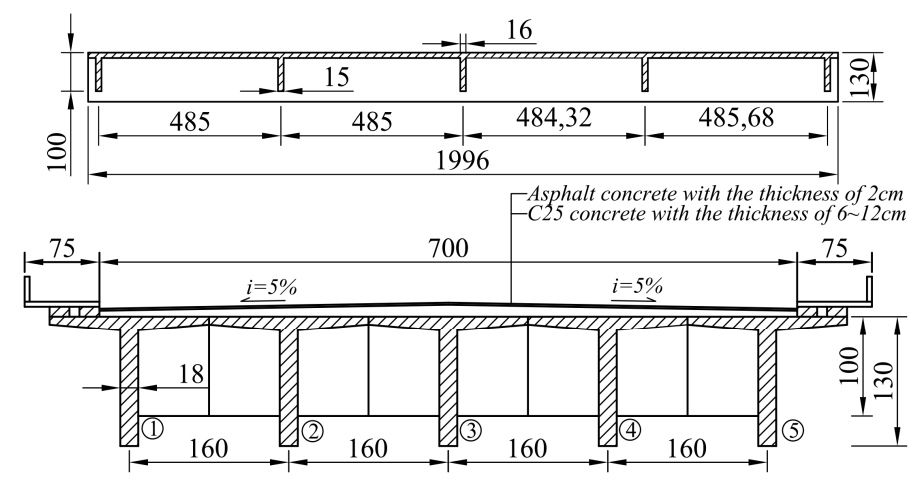

Figure 2. Bridge layout (units: $\mathrm{cm}$ )

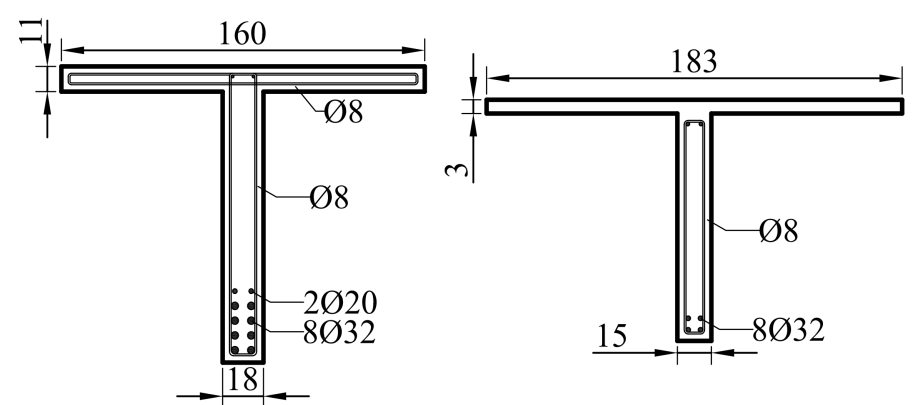

Figure 3. Cross section and reinforcement (units: $\mathrm{cm}$ )

The finite element model is established for this bridge, including 65 nodes and 80 grid elements. Each node has 3 DOFs corresponding to the force resultant: bending, torsion and shear. 

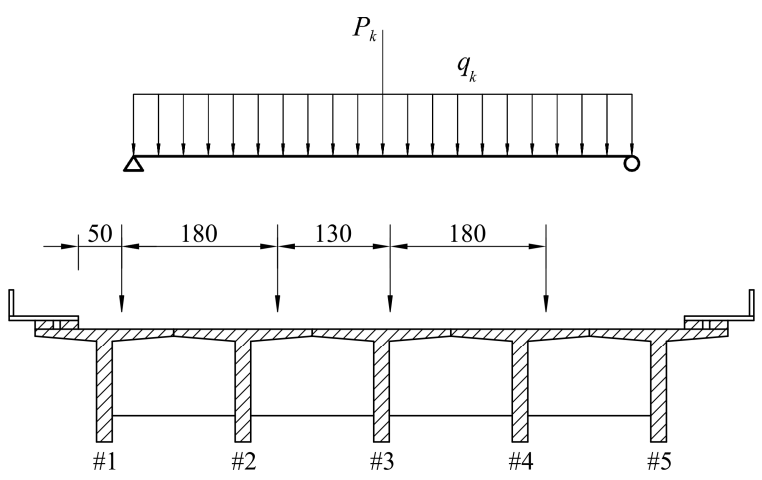

Figure 4 Live load position in two directions

The loads considered are dead load, vehicle load, and impact loads for a highway bridge. Dead load is modeled as the uniformly distributed force along the bridge. The nominal RC gravity density is set to be $25.0 \mathrm{kN} / \mathrm{m}^{3}$ for self-weight calculation. The vehicle load is simplified to be the combination of a uniformly distributed force with the magnitude of $q_{k}$ and a concentrated force with the magnitude of $P_{k}$. According to Chinese GB/T 50283-1999 code, $q_{k}$ is set to be $7.785 \mathrm{kN} / \mathrm{m}$ and $P_{k}$ is $178.5 \mathrm{kN}$, respectively. The worst load position is specified by G-M method in two directions as shown in Figure 4. The impact factor is 0.2695 , calculated according to the fundamental frequency of this bridge.

\section{COMPONENT AND BRIDGE RESISTANCE}

The calculation of the ultimate component section resistance is conducted according to Chinese JTG D62-2004 code, US ACI 318-11 code, and EPT model. In this study, the shear resistance of the compression flanges is not considered. More detailed investigations to account for this effect can reference the work by Schütte and Sigrist (2014).

Table 1: Shear capacity (units: $\mathrm{kN}$ )

\begin{tabular}{lllll}
\hline Section & Chinese Code & ACI Code & Mode t & Mode s \\
\hline Girder (near hearings) & 563.3 & 442.0 & 577.7 & 419.9 \\
Girder (mid-span) & 398.3 & 301.0 & 408.5 & 296.9 \\
Diaphragm & 282.6 & 254.3 & 177.0 & 143.8 \\
\hline
\end{tabular}

Table 2: Torsional capacity (units: $\mathrm{kNm}$ )

\begin{tabular}{|c|c|c|c|c|}
\hline Section & Chinese Code & ACI Code & Mode $\mathrm{t}$ & Mode s \\
\hline Girder (near hearings) & 56.7 & 42.0 & 67.6 & 50.7 \\
\hline Girder (mid-span) & 43.9 & 21.0 & 47.8 & 35.9 \\
\hline
\end{tabular}

Table 3: Flexural capacity (units: $\mathrm{kNm}$ )

\begin{tabular}{llll}
\hline Section & Chinese Code & ACI Code & FPT model \\
\hline Firder & 27367 & 27367 & 27367 \\
Diaphragm & 395.4 & 395.4 & 395.4 \\
\hline
\end{tabular}

The bridge failure mode and corresponding position can be computed based on the static incremental method on the FEM model. The bridge generalized resistance is defined as $\lambda$ times standard vehicle load considering impact effect. First failure occurs at $1 / 4$ span of side girder (Figure 5) with the generalized resistance $\lambda=1.5438$ controlled by shear-torsion dominated failure mode $\mathrm{s}$. 
Table.4 Bridge first cross-section failure and generalized resistance $\lambda$

\begin{tabular}{lll}
\hline Rules & $\lambda$ & Failure mode \\
\hline JTG D62-2004 code & 2.3297 & Pure bending \\
ACI 318-11 code & 2.3297 & Pure beinding \\
JTG D62-2004 code & 2.8215 & Pure shear \\
ACI 318-11 code & 1.8278 & Pure shear \\
JTG D62-2004 code & 3.2145 & Pure torsion \\
ACI 318-11 code & 2.2915 & Pure torsion \\
EPT model & 1.8709 & Mode t \\
EPT model & 1.5438 & Mode s \\
\hline
\end{tabular}

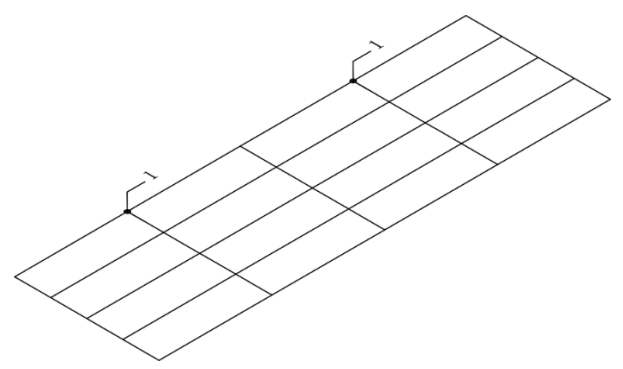

Figure 5: Bridge first cross-section failure position (mode s)

To avoid mode $\mathrm{s}$ failure, the stirrup spacing at the $1 / 8$ span of girder is adjusted. The relationship between $s_{1 / 8}$ and $\lambda$ is shown in Figure 7 , as the variation of $\lambda$ the failure mode and corresponding position also changes. The critical spacing to determine whether the first failure is ductile is about $150 \mathrm{~mm}$, in this study, $s_{1 / 8}$ is set to be $100 \mathrm{~mm}$ for the robustness of ductile failure mode.

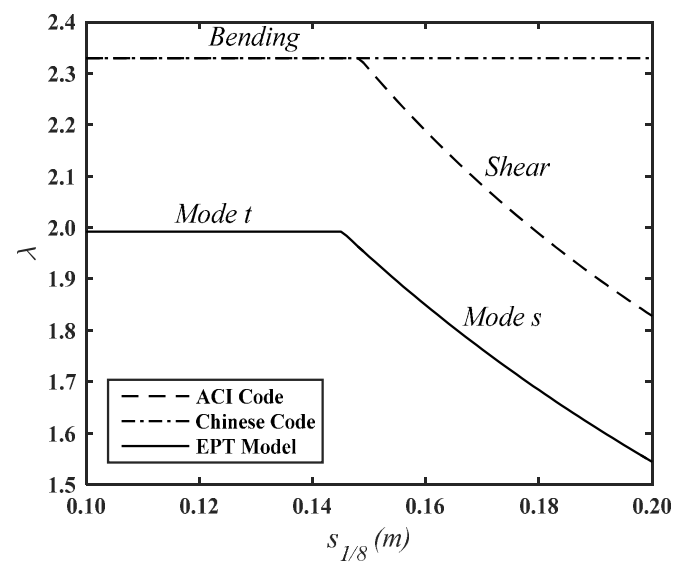

Figure 6. Relationship between $\lambda$ and $s_{1 / 8}$

After the adjustment of stirrup spacing $s_{1 / 8}$, value of $\lambda$ for pure shear failure increases significantly. The value of $\lambda$ for pure torsion failure has almost no change, as shown in table 5 . It indicates that shear-torsion failure is dominated by shear force. In addition, the increase of $\lambda$ for failure mode $t$ is smaller than $\lambda$ for failure mode $s$. The generalized resistance becomes the one controlled by ductile bending failure according to EPT model theory. The failure occurs at the midspan of side girder (Figure 8) and $\lambda=1.9919$.

Table.5 Bridge first failure and generalized resistance $\lambda$ after stirrup adjustment 


\begin{tabular}{lll}
\hline Rules & $\lambda$ & Failure mode \\
\hline Chinese Code (D62) & 2.3297 & Pure bending \\
ACI Code & 2.3297 & Pure bending \\
Chinese Code (D62) & 3.6500 & Pure shear \\
ACI Code & 2.5505 & Pure shear \\
Chinese Code (D62) & 3.2145 & Pure torsion \\
ACI Code & 2.4503 & Pure torsion \\
Equilibrium truss model & 1.9919 & Mode t \\
Equilibrium truss model & 2.2186 & Mode s \\
\hline
\end{tabular}

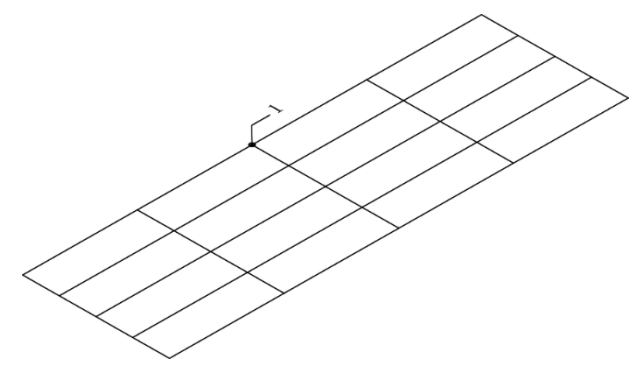

Figure 7. Bridge first cross-section failure (mode t) position after stirrup adjustment

\section{SENSITIVITY ANALYSIS}

The bridge structure capacity is related with resistance and load parameters, the sensitivity analysis on which could find the most sensitive parameters to bridge performance. The parameters to be studied in this paper are girder web thickness $b$, diaphragm web thickness $b_{d}$, concrete strength $f_{c}$, rebar area $A_{s}$, stirrup area $A_{w}$, rebar strength $f_{s}$, stirrup strength $f_{w}$, girder effective depth $h_{0}$ and diaphragm effective depth $h_{d 0}$. It is shown in Figure 8 that the diaphragm web thickness, girder effective depth, rebar area and strength are the significant influential structural parameters on bridge load carrying capacity.

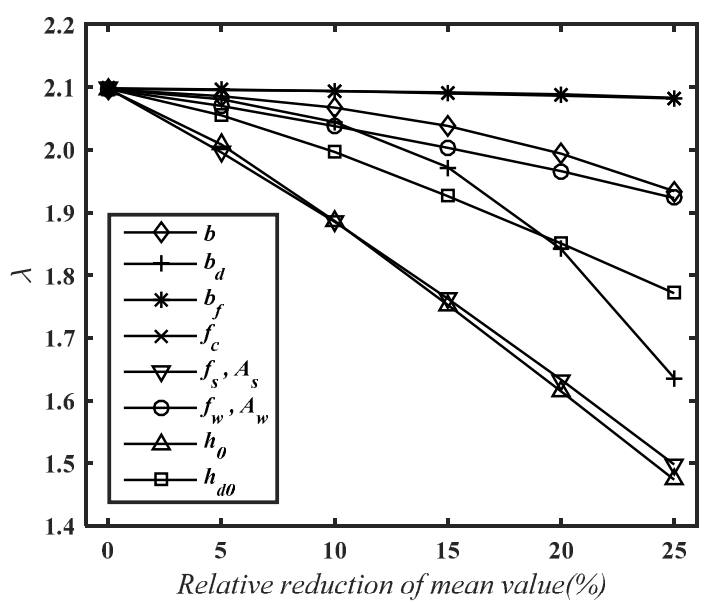

Figure 8: $\lambda$ variation considering the reduction of parameters

\section{CONCLUSIONS}

Considering the B-T-S interaction, this paper conducts the assessment of the first cross-section failure of a simply supported reinforced concrete grillage bridge under vehicle lane load. The result in- 
dicates that 1) Interaction of bending, torsion and shear will reduce the flexural capacity of RC grid components. 2) Shear or torsion failure may occur before the designed girder ductile bending failure, there is a risk of uncontrollable failure for simply supported grillage bridge. 3) Girder effective depth is the most influential factor on reliability of simply-supported grillage bridge in combined BT-S interaction.

\section{ACKNOWLEDGEMENT}

This research is supported by the 973 program of Ministry of Science and Technology of China (Grant No. 2013CB036305).

\section{REFERENCES}

1) American Concrete Institutes. 2011. Building Code and Requirements for Structural Concrete (ACI 318-11) and Commentary. Farmington Hills.

2) Xu, D., Zhao, Y., \& Liu, C. 2014. Experimental study on shear behavior of reinforced concrete beams with web horizontal reinforcement [J]. Front. Struct. Civ. Eng., 8(4): 325-336.

3) Ewida, A. A. \& McMullen, A. E.1981. Torsion-shear-flexure interaction in reinforced concrete members [J]. Magazine of Concrete Research. (33)115:113-122.

4) Elfgren, L. 1972. Reinforced concrete beams loaded in combined torsion, bending and shear - a study of the ultimate load-carrying capacity. Publications 71:3. Division of Concrete Structures, Chalmers University of Technology, Goteborg, Sweden.

5) Hsu, T. T. C. \& Mo, Y. L. 2010. Unified theory of concrete structures. Wiley, New York.

6) Ministry of Communication of People's Republic of China. 2004. General Code for Design of Highway Bridges and Culverts (JTG D60 2004). Beijing, China Communications Press.

7) Ministry of Communication of People's Republic of China. 1999. Unified Standard for Reliability Design of Highway Engineering Structures (GB/T 50283-1999). Beijing, China Planning Press.

8) Schütte, B. \& Sigrist, V. 2014. 7 [J]. Shear assessment of compression flanges of structural concrete T-beams. Front. Struct. Civ. Eng., 8(4): 354-361.

9)Zhang, Y., Sun, Z., \& An, Y. 2014. Vehicle carrying safety assessment for highway grillage narrow bridge considering lumped plasticity and engineering uncertainty [C]. Proceeding of IABMAS, Shanghai, China. 